\title{
O CONHECIMENTO, A VALORIZAÇÃO DA TRIAGEM AUDITIVA NEONATAL E A INTERVENÇÃO PRECOCE DA PERDA AUDITIVA
}

\section{The knowledge and valorization of neonatal auditory screening and the early intervention of hearing loss}

\author{
Maria Regina Pereira Boeira Hilú ${ }^{(1)}$, Bianca Simone Zeigelboim ${ }^{(2)}$
}

\begin{abstract}
RESUMO
Objetivo: investigar o conhecimento, a valorização da detecção precoce da deficiência auditiva pelos profissionais da saúde envolvidos no período pré e pós gestacional e verificar o conhecimento das mães sobre a importância da triagem auditiva neonatal. Métodos: o instrumento da pesquisa constituiu-se de três questionários com perguntas fechadas e específicas aos profissionais envolvidos; pediatras, ginecologistas/obstetras e enfermeiros, além das mães dos bebês nascidos em uma maternidade na cidade de Maringá/PR, no período de janeiro a fevereiro/2005. Resultados: observou-se no questionário realizado com os médicos que todos, $100 \%$, referiram ter conhecimento da saúde auditiva do bebê, $69 \%$ recomendaram o teste da orelhinha, $82 \%$ referiram que até os primeiros três meses seria o período ideal para o diagnóstico auditivo e apenas $37 \%$ souberam referir quais os exames necessários a serem realizados. Entre os enfermeiros, $78 \%$ demonstraram ter conhecimento sobre a detecção precoce da perda auditiva e $67 \%$ referiram orientar os pais sobre o teste da orelhinha. Sobre a importância da detecção precoce da deficiência auditiva, $22 \%$ não responderam ou não acharam relevante. No questionário realizado com as mães, $81 \%$ relataram não terem recebido orientações a respeito das doenças que alteram o desenvolvimento auditivo, $72 \%$ não tinham conhecimento a respeito da detecção precoce da deficiência auditiva. Conclusão: mostrou-se evidente neste estudo um conhecimento reduzido com relação a detecção precoce da perda auditiva e da triagem auditiva neonatal por parte das pessoas envolvidas nesta pesquisa.
\end{abstract}

DESCRITORES: Triagem Neonatal; Audição; Perda Auditiva

\section{INTRODUÇÃO}

A audição é essencial para a aquisição da linguagem oral, uma vez que é pela interação com o outro que já detém linguagem, que a criança consegue entender seu universo, compreender seus semeIhantes, desenvolver e organizar pensamentos e sentimentos e adquirir conhecimento. Do ponto de vista intelectual, a audição é o órgão mais importante ${ }^{1}$. No final do sexto mês gestacional, o aparelho auditivo está completamente formado e funcionando

(1) Fonoaudióloga; Mestre em Distúrbios da Comunicação pela Universidade Tuiuti do Paraná.

(2) Fonoaudióloga; Coordenadora do Programa de Mestrado e Doutorado em Distúrbios da Comunicação da Universidade Tuiuti do Paraná; Doutora em Ciências dos Distúrbios da Comunicação Humana pela Universidade Federal de São Paulo. adequadamente, o que vem confirmar a sua importância para o desenvolvimento neuro-psicomotor da criança ${ }^{2}$.

Para o seu desenvolvimento é de suma importância a maturação da via auditiva, que depende da qualidade "comunicativa e auditiva" a que a criança está exposta. O componente neural irá se desenvolver a partir das experiências vividas pela criança, pois é nesse período (até aproximadamente dois anos) que se dá a plasticidade auditiva ${ }^{3}$. Em função disso, o diagnóstico audiológico realizado durante o primeiro ano de vida possibilita a intervenção, médica e/ou fonoaudiológica, ainda no período crítico de maturação e plasticidade funcional do sistema nervoso central, permitindo um prognóstico favorável em relação ao desenvolvimento global da criança ${ }^{3}$.

Prevenir a perda auditiva é uma forma de proteger e impedir que a criança sofra os efeitos funestos provocados pela falta de estimulação auditiva sobre 
a função da linguagem ${ }^{4}$. Cerca de $50 \%$ das perdas auditivas poderiam ser evitadas ou suas seqüelas diminuídas, se ocorressem precocemente medidas de detecção, diagnóstico e reabilitação ${ }^{5}$.

A Academia Americana de Pediatria revela que a deficiência auditiva permanente atinge de um a três em 1000 bebês nascidos e acompanhados em berçários normais e de dois a quatro bebês, em um grupo de 1000 nascimentos acompanhados em UTI'S neonatais. Afetam também crianças na idade pré-escolar, em decorrência de doenças como meningite e otite média secretora adquirida antes dos primeiros doze meses de vida ${ }^{6}$.

A prevalência de deficiência auditiva é vinte vezes maior que outras doenças como a fenilcetonúria ou hipotireoidismo e sua identificação demanda um custo dez vezes menor que para outras doenças ${ }^{2}$.

O papel do pediatra é imprescindível para que os programas de detecção e intervenção precoces da audição sejam bem sucedidos, porém há necessidade do envolvimento de toda a equipe de enfermagem, a família e a comunidade ${ }^{7,8}$.

O processo de identificação precoce da deficiência auditiva deve ser iniciado ainda no berçário, através da triagem auditiva neonatal (TAN) por ser uma forma eficiente de identificar, principalmente, as crianças de risco. No entanto, a triagem por si só não traz nenhum benefício para a saúde da criança, simplesmente identifica. O correto seria, logo após o nascimento, identificar as crianças de risco, encaminhar para confirmação da suspeita da deficiência auditiva e enquadrar em processos terapêuticos ${ }^{9}$.

O Comitê Brasileiro sobre Perdas Auditivas na Infância (CBPAl) ${ }^{10}$ recomenda a implantação da triagem auditiva neonatal universal (TANU) para todas as crianças do nascimento até os três meses de idade. $O$ Joint Committee on Infant Hearing $(\mathrm{JClH}){ }^{11}$ recomenda que todo o recém nascido deve ter sua audição avaliada, tendo em vista a grande incidência de alterações em bebês que não estão inseridos em um grupo indicador de risco. Nos casos de deficiência auditiva confirmada, deve haver intervenção educacional até os seis meses de idade.

A Academia Americana de Pediatria recomenda a utilização de métodos eletrofisiológicos nos programas de TAN como o potencial evocado auditivo de tronco encefálico (PEATE) e as emissões otoacústicas evocadas (EOAE) (Teste da Orelhinha) ${ }^{5}$. Para que se possa fazer o diagnóstico precoce, os bebês considerados de risco para deficiência auditiva ou não, devem realizar a TAN nas primeiras 48 horas de vida ou antes da alta hospitalar ${ }^{11}$.

O PEATE avalia a integridade neural das vias auditivas até o tronco encefálico, através do registro das ondas eletrofisiológicas, geradas em resposta a um som apresentado e captado por eletrodos de superfície colocados na cabeça. As EOAE registram a energia sonora gerada pelas células ciliadas da cóclea, em resposta aos sons apresentados e gravados por microfone miniaturizado colocado no conduto auditivo externo ${ }^{10}$. Os métodos citados são rápidos, não invasivos e de fácil aplicação. Não sendo possível realizar a triagem pelos métodos eletrofisiológicos é possível investigar junto aos fatores de risco, a observação do comportamento auditivo e pesquisa do reflexo cócleo-palpebral, porém perdas leves ou unilaterais não podem ser diagnosticadas por este método ${ }^{10}$.

É fundamental o conhecimento e a valorização de todos os profissionais de saúde envolvidos no período pré e pós gestacional para que haja efetividade nos programas de triagem auditiva, tendo em vista a detecção precoce da perda auditiva por meio do teste da orelhinha. Todo o esforço só é válido se existir a consciência imediata dos profissionais que atuam diretamente com os bebês, com o intuito de dar início ao processo de (re)habilitação.

$\mathrm{O}$ objetivo do presente estudo foi investigar o conhecimento, a valorização da detecção precoce da deficiência auditiva pelos profissionais da saúde envolvidos no período pré e pós gestacional e verificar o conhecimento das mães sobre a importância da TAN.

\section{MÉTODOS}

Trata-se de um estudo transversal observacional que envolveu médicos pediatras, ginecologistas/obstetras e enfermeiros, inseridos no setor de neonatologia, além das mães dos bebês nascidos em uma maternidade na cidade de Maringá/PR no período de janeiro a fevereiro/2005.

Essa maternidade realiza em torno de quarenta nascimentos mês e recebe um público de convênios, particulares e do Sistema Único de Saúde (SUS).

Os dados foram analisados de modo quantitativa e qualitativa sendo utilizado como instrumento de coleta de dados três questionários elaborados pelo núcleo de pesquisa "Percepção Auditiva" da Universidade Tuiuti do Paraná contendo perguntas fechadas, específicas para cada área profissional envolvida, além das mães dos bebês.

O critério de inclusão na pesquisa foi que os profissionais de saúde fizessem parte do corpo clínico da maternidade.

Iniciou-se a pesquisa com o esclarecimento do objetivo, seguido pela entrevista com cada profissional. Aos médicos (pediatras e ginecologistas/ obstetras) foram enviados 30 questionários com nove perguntas e 16 retornaram devidamente preenchidos (Figura 1). Aos enfermeiros foram envia- 
dos 12 questionários com sete perguntas e deste houve a devolução de nove (Figura 2).

Com relação as mães, os critérios de inclusão foram: a) a aplicação do questionário com 11 perguntas deveria ocorrer antes da apresentação e explicação sobre o programa de TAN; b) as questões deveriam ser respondidas nas primeiras 48 horas após o parto, ou antes da alta hospitalar; c) as mães deveriam apresentar condições físicas e emocionais para participar da pesquisa. Participaram da amostra 32 sujeitos (Figura 3).

Os dados estatísticos da pesquisa foram catalogados com o auxílio do software Sphinx Léxica e analisados de modo qualitativo e quantitativo.

Ressalta-se que o estudo foi aprovado pelo Comitê de Ética em Pesquisa da Universidade Tuiuti do Paraná, parecer no 48/2004 e os participantes colaboraram espontaneamente, sendo respeitados todos os preceitos éticos inerentes à pesquisa.

\section{RESULTADOS}

\section{Resultados da pesquisa realizado com os médicos}

A porcentagem de respostas obtidas foi de $53 \%$. Não houve distinção em relação ao sexo e tempo de formação, tendo a maioria dos entrevistados entre 15 e 20 de formados. Quanto ao conhecimento a respeito da detecção precoce da deficiência auditiva, $100 \%$ dos profissionais responderam ter conhecimento e acharam relevante investigar a audição, principalmente, em crianças que apresentam fatores de risco. Destes, $56 \%$ afirmaram orientar as famílias a respeito da saúde auditiva do bebê durante o pré-natal ou na visita ao pediatra e $44 \%$ responderam que não o fazem.

Com relação ao "teste da orelhinha", $69 \%$ dos médicos recomendam os pais a realizarem o teste $e$ $31 \%$ não os orientam. Houve unanimidade, ou seja, $100 \%$ dos médicos acharam importante que, qualquer criança que nasça com algum fator de risco para a perda auditiva deva ser investigada. Quando a criança não apresenta nenhum fator de risco, apenas $13 \%$ dos médicos (ginecologistas/obstetras) não acharam relevante a sua investigação. Apesar de $100 \%$ dos médicos acharem relevante a investigação a respeito da deficiência auditiva em neonatos com e sem fatores de risco, $56 \%$ dos entrevistados desconhecem que $50 \%$ dos bebês com perda auditiva não fazem parte do grupo de risco.

Referente a idade ideal para a realização do diagnóstico audiológico, $82 \%$ dos entrevistados responderam ser possível nos primeiros três meses, $12 \%$ em outras idades e 6\% não responderam. Quanto as técnicas utilizadas para a realização da avaliação auditiva, $63 \%$ afirmaram conhecer, porém não sou- beram descrevê-las, $25 \%$ responderam o exame de EOA, 6\% responderam avaliação comportamental e $6 \%$ o exame de PEATE. Observou-se nos profissionais envolvidos na pesquisa, pouco conhecimento a respeito dos testes que fazem parte da TAN.

\section{Resultados da pesquisa realizado com os enfermeiros}

O índice de respostas obtidas foi de $75 \%$ e o tempo de formação foi de mais de cinco anos. Setenta e oito por cento dos enfermeiros responderam ter conhecimento sobre a detecção precoce da deficiência auditiva. Com relação ao "teste da orelhinha", $67 \%$ referiram orientar os pais sobre a importância e necessidade do procedimento, $22 \%$ não orientam e $11 \%$ não responderam.

Todos os enfermeiros entrevistados acharam importante investigar a deficiência auditiva em crianças com e sem fatores de risco. Apesar disso, $100 \%$ desconhecem que apenas $50 \%$ das crianças com deficiência auditiva não fazem parte do grupo de risco.

Para conhecer o nível de consciência dos enfermeiros sobre a importância da detecção precoce da deficiência auditiva, foi perguntado a eles qual a idade ideal para realizar o diagnóstico da audição. Cinquenta e seis por cento responderam nos primeiros três meses, $22 \%$ em outras idades, $11 \%$ não responderam e $11 \%$ não acharam relevante.

\section{Resultado da pesquisa realizada com as mães}

Das 32 mães que participaram do estudo, todas realizaram pré-natal mensalmente, $69 \%$ em clínicas particulares e $31 \%$ em postos de saúde.

Com relação a orientação a respeito de doenças que alteram o desenvolvimento auditiva da criança, $26(81 \%)$ disseram não terem recebido orientação enquanto seis (19\%) relataram terem sido orientadas. Dessas, três por meio médico e as demais por meio de parentes, panfletos e televisão.

Com relação ao conhecimento da detecção precoce da deficiência auditiva, $72 \%$ das mães referiram não ter conhecimento e $28 \%$ disseram que sim. Destas, $13 \%$ através de orientação médica, $2 \%$ por fonoaudiólogos e $13 \%$ por veículos de comunicação.

Apesar de $56 \%$ das mães responderem que saberiam reconhecer se o seu filho ouve bem $75 \%$ responderam não ter conhecimento a respeito do desenvolvimento da audição e linguagem da criança. Diversas mães comentaram que já por terem outros filhos, conseguiriam identificar o dano auditivo, por comparação entre irmãos. Observa-se que $69 \%$ das mães gostariam de obter informações a respeito do desenvolvimento da audição e linguagem, $22 \%$ não responderam e $9 \%$ responderam que não teriam interesse. 
1. Há quanto tempo você é formado?
( ) menos de 1 ano
( ) 1 a 5 anos
( ) 5 a 10 anos
( ) 10 a 15 anos
( ) 15 a 20 anos
( ) mais de 20 anos

2. Você tem conhecimento a respeito da detecção precoce da deficiência auditiva?

$$
\text { ( ) sim }
$$

3. Durante as visitas do pré-natal ou durante uma visita ao pediatra, você orientou seu paciente a respeito da saúde auditiva do bebê?

$$
\text { ( ) sim }
$$

4. Você orientou os pais a realizarem o teste da orelinha?

$$
\text { ( ) sim }
$$

5. Você acha relevante investigar a deficiência auditiva em crianças que não apresentam fatores de risco?

$$
\text { ( ) sim }
$$

6. Você acha relevante investigar a deficiência auditiva em crianças que apresentam fatores de risco?

$$
\text { ( ) sim }
$$

7. Você tem conhecimento que $50 \%$ das crianças com perda auditiva não fazem parte do grupo de risco para deficiência auditiva?

$$
\text { ( ) sim }
$$

8. Qual idade você julga ideal para realizar o diagnóstico de perda auditiva na infância?
( ) até 3 meses
( ) até 6 meses
( ) até 1 ano
( ) até 2 anos
( ) até 3 anos
( ) até 4 anos
( ) não acha a idade relevante

9. Na sua opinião, qual dessas técnicas é a mais fidedigna para avaliar a audição do recém-nascido?
( ) BERA - Audiometria de Tronco Encefálico
( ) Avaliação Comportamental
( ) EOA - Emissões Otoacústicas

\section{Figura 1 - Questionário realizado com os médicos}


1. Há quanto tempo você é formado?
( ) menos de 1 ano
( ) 1 a 5 anos
( ) 5 a 10 anos
( ) 10 a 15 anos
( ) 15 a 20 anos
( ) mais de 20 anos

2. Você tem conhecimento a respeito da detecção precoce da deficiência auditiva?

$$
\begin{aligned}
& \text { ( ) sim } \\
& \text { ( ) não }
\end{aligned}
$$

3. Você orientou os pais a realizarem o teste da orelhinha?

$$
\begin{aligned}
& \text { ( ) sim } \\
& \text { ( ) não }
\end{aligned}
$$

4. Você acha relevante investigar a deficiência auditiva em crianças que não apresentam fatores de risco?

$$
\text { ( ) sim }
$$

5. Você acha relevante investigar a deficiência auditiva em crianças que apresentam fatores de risco?

$$
\begin{aligned}
& \text { ( ) sim } \\
& \text { ( ) não }
\end{aligned}
$$

6. Você tem conhecimento que $50 \%$ das crianças com perda auditiva não fazem parte do grupo de risco para deficiência auditiva?
( ) $\operatorname{sim}$
( ) não

7. Qual idade você julga ideal para realizar o diagnóstico de perda auditiva na infância?
( ) até 3 meses
( ) até 6 meses
( ) até 1 ano
( ) até 2 anos
( ) até 3 anos
( ) até 4 anos
( ) não acha a idade relevante

Figura 2 - Questionário realizado com os enfermeiros 
1. Você fez consultas pré-natais?

$$
\begin{aligned}
& \text { ( ) sim } \\
& \text { ( ) não }
\end{aligned}
$$

2. Se sim, onde?

$$
\text { ( ) clínica particular }
$$

3. Fez consultas ao médico?
( ) mensalmente
( ) bimestralmente
( ) trimestralmente

4. Durante a gestação você foi orientada sobre doenças que poderiam alterar o desenvolvimento auditivo do seu bebê?

$$
\begin{aligned}
& \text { ( ) sim } \\
& \text { ( ) não }
\end{aligned}
$$

5. Se sim, quem orientou?

$$
\begin{aligned}
& \text { ( ) médico } \\
& \text { ( ) fonoaudiólogo } \\
& \text { ( ) enfermeira } \\
& \text { ( ) outros } \\
& \text { especifique }
\end{aligned}
$$

6. Você já ouviu falar em detecção precoce da deficiência auditiva?

$$
\begin{aligned}
& \text { ( ) sim } \\
& \text { ( ) não }
\end{aligned}
$$

7. Se sim, por qual meio?
( ) orientação médica
( ) orientação fonoaudióloga
( ) pelos atendentes da maternidade
( ) meios de comunicação

8. Você pensou a respeito da saúde auditiva do seu bebê durante a gestação?

$$
\text { ( ) sim }
$$

9. Você acha que saberia reconhecer se seu filho ouve ou não?

$$
\text { ( ) sim }
$$

10. Você tem conhecimento a respeito do desenvolvimento de audição e linguagem?

$$
\begin{aligned}
& \text { ( ) sim } \\
& \text { ( ) não }
\end{aligned}
$$

11. Se não, gostaria de receber tais informações?

$$
\begin{aligned}
& \text { ( ) sim } \\
& \text { ( ) não }
\end{aligned}
$$

Figura 3 - Questionário realizado com as mães 


\section{DISCUSSÃO}

A deficiência auditiva é uma patologia que a literatura reconhece como grave, uma vez que pode apresentar conseqüências importantes no desenvolvimento cognitivo, social, intelectual e da linguagem, dos indivíduos portadores da surdez quando não diagnosticada no tempo correto. A integridade do aparelho auditivo para o desenvolvimento neuropsicomotor da criança é de extrema importância. $O$ componente neural irá se desenvolver até aproximadamente dois anos, sendo este o tempo de ocorrência da plasticidade auditiva ${ }^{2,3}$.

A literatura indica a TANU a todos os recém-nascidos, independente de fazerem ou não parte do grupo de risco para a deficiência auditiva. Tais fatores de riscos foram descritos pelo Joint Committee on Infant Hearing $(\mathrm{JClH}){ }^{10-12}$ e pelo autor ${ }^{2}$. Os profissionais de saúde envovidos na presente pesquisa apresentaram ter pouco conhecimento a respeito da TANU. $\mathrm{O} \mathrm{JCIH}{ }^{10}$ e o grupo de apoio a triagem auditiva neonatal universal (GATANU) afirmam que todo recém-nascido deve ter sua audição avaliada, tendo em vista a grande incidência do problema em bebês que não apresentaram intercorrências pré, peri ou pós-natal.

Sobre a idade ideal para o diagnóstico da deficiência auditiva, a literatura expõe que o primeiro ano de vida é importantíssimo para a descoberta da surdez, uma vez que possibilita a intervenção médica e (ou) fonoaudiológica ainda no período crítico da maturação das vias auditivas. Na pesquisa realizada, $82 \%$ dos médicos e $56 \%$ dos enfermeiros acreditam que o diagnóstico da deficiência auditiva deva ser realizada até os três meses de idade, entendendo assim que a intervenção deva ocorrer até os seis meses concordando com diversos estu$\operatorname{dos}^{1,3,13}$.

Quando questionado aos médicos sobre qual técnica seria a mais fidedigna para avaliar a audição dos bebês, verificou-se que $63 \%$ afirmaram conhecer porém não souberam descrevê-las, provavelmente por falta de conhecimento dos exames. O autor ${ }^{14}$ observou que apenas uma minoria, 13,5\% dos médicos entrevistados, referiram as EOA como sendo o exame mais recomendado a ser realizado nos bebês. No presente estudo, $25 \%$ dos médicos referiram-se as EOA como sendo o melhor exame para identificação da perda auditiva.

Estudos realizados ${ }^{8,15}$ apontaram que há necessidade de mais informações sobre a importância do diagnóstico precoce, dos métodos para sua realização e que o conhecimento é restrito no que diz respeito à deficiência auditiva para toda a equipe envolvida no período pré e pós gestacional.

Observou-se uma contradição entre as informações dadas pelos profissionais de saúde envolvidos no estudo e as mães entrevistadas. Os profissionais relataram conhecer a detecção precoce da deficiência auditiva e orientar sobre a TAN, enquanto que as mães relataram, em sua maioria, não terem tido acesso a nenhum tipo de informação sobre o desenvolvimento auditivo do bebê durante a gestação e de sua permanência na maternidade. Contradição importante de ser considerada e refletida. Esses resultados estão em acordo ${ }^{7}$ que observou em seus estudos que $78 \%$ das mães entrevistadas não tinham conhecimento algum.

\section{CONCLUSÃO}

Mesmo tratando-se de uma amostra restrita, concluiu-se a necessidade de informações sobre a importância do diagnóstico precoce, bem como, dos métodos utilizados para a sua realização por parte dos profissionais da saúde envolvidos na presente pesquisa. Este fato talvez ocorra por várias razões, dentre elas, evidencia-se: falhas no processo de graduação dos profissionais, falta de interesse e desatualização dos mesmos e pouco incentivo do governo e das instituições devido a falta de uma política pública clara e eficiente sobre o problema.

Uma forma de tentar diminuir o problema, seria oferecer aos profissionais de saúde envolvidos no período pré e pós gestacional maiores informações a respeito e conseqüentemente esses poderiam orientar melhor as famílias por meio de campanhas e cursos nas maternidades e postos de saúde do Brasil sobre a importância da realização do teste da orelinha já nas primeiras 48 horas de vida do bebê. 


\begin{abstract}
Purpose: to investigate the knowledge and valorization of early detection of hearing loss by health professionals involved in the period before and after gestation and to check the mothers' knowledge on the importance of neonatal auditory screening. Methods: the research instrument consisted of three questionnaires with closed and specific questions for the involved professionals, including pediatricians, gynecologists, obstetricians and nurses, in addition to the mothers of newborns from a maternity ward in the city of Maringá, Paraná, during the period between January to February 2005. Results: it was observed in the questionnaire completed with the doctors that $100 \%$ described having knowledge of the baby's auditory health, $69 \%$ recommended otoacoustic emissions (OAE) $82 \%$ related that within the first three months it would be the ideal period for an auditory diagnosis and just $37 \%$ knew which were the exams to be performed. Among the nurses, $78 \%$ demonstrated having knowledge on early detection of hearing loss and $67 \%$ related having trained the parents to perform the (OAE). With regard to the importance of early detection of hearing loss, $22 \%$ did not answer or did not find it important. In the questionnaire completed with the mothers, $81 \%$ described not having received counseling regarding the diseases that alter auditory development, $72 \%$ did not have any knowledge regarding early detection of hearing loss. Conclusion: a reduced knowledge was found to be evident in this study, with regard to the early detection of hearing loss and neonatal auditory screening, by the people involved in this research.
\end{abstract}

KEYWORDS: Neonatal Screening; Hearing; Hearing Loss

\section{REFERÊNCIAS}

1. Roslyng-Jensen AMA. Importância do diagnóstico precoce na deficiência auditiva. In: Lopes Filho $\mathrm{O}$, organizador. Tratado de fonoaudiologia. São Paulo: Roca; 1997. p. 297-309.

2. Nobrega M. Triagem audiológica universal. In: Caldas Neto S, Sih T. Otologia e audiologia em pediatria. Rio da Janeiro: Revinter; 1999. p. 208-10. 3. Azevedo MF. Avaliação audiológica no $1 .^{\circ}$ ano de vida In: Lopes Filho O, organizador. Tratado de fonoaudiologia. São Paulo: Roca; 1997. p. 604-16.

4. Russo ICP, Santos TMM. Audiologia infantil. São Paulo: Cortez; 1994.

5. Lewis DR, Costa Filho OA. Surdez no recém-nascido. In: Campos $\mathrm{CAH}$, Costa HOO, organizadores. Tratado de otorrinolaringologia. São Paulo: Roca; 2003. p. 367-78.

6. Jensen AMAR, Fragoso ACPF. Reabilitação da perda auditiva na infância. In: Campos $\mathrm{CAH}$, Costa $\mathrm{HOO}$, organizadores. Tratado de otorrinolaringologia. São Paulo: Roca; 2003. p. 232-42.

\section{RECEBIDO EM: 02/07/2007}

ACEITO EM: 05/11/2007

Endereço para correspondência:

Rua Gutenberg, 99 9a andar

Curitiba - PR

CEP: 80420-030

Tel/Fax: (41) 33317807

E-mail: bianca.zeigelboim @utp.br
7. Ribeiro FG, Miltre El. Avaliação do conhecimento sobre triagem auditiva neonatal de pacientes no pós-parto imediato. Rev CEFAC. 2004; 6(3):294-9.

8. Zocoli AM, Ricchel FC, Zeigelboim BS, Marques JM. Audição: abordagem do pediatra acerca dessa temática. Rev Bras Otorrinolaringol. 2006; 72(5):617-23.

9. Weiss KM. Pediatras e neonatologistas: detecção precoce da deficiência auditiva. [monografia]. Porto Alegre (RS): CEFAC - Saúde e Educação; 1999.

10. Comitê Brasileiro de Perda Auditiva na Infância. Recomendações n.01/99; 2000 do Comitê Brasileiro de Perda Auditiva na Infância. J Cons Fed Fonoaudiol. 2000; 5:3-7.

11. Joint Committee on Infant Hearing. Position statement: principles and guidelines for early hearing detection and intervention programs. Am J Audiol. 2000; 9(1):9-29.

12. Joint Committee on Infant Hearing. Joint Committee on Infant Hearing 1994 Position statement . Pediatrics. 1995; 95(1):152-6.

13. Yoshinaga-Itano C, Stredler-Brown A, Mehl AL. Language of early and later identification and early intervention. In: Seminars in Hearing. New York: Thième; 1998.

14. Rabelo BGR, Salomão LM, Carnivali PA, Leite ICG. Algumas considerações sobre o grau de conhecimento dos pediatras sobre questões fonoaudiológicas. Fono Atual. 2004; 27(7):4-101.

15. Barros ACT, Galindo MAC, Jacob RTS. Conhecimento e conduta de pediatras frente à deficiência auditiva. Pediatria. 2002; 24(1):25-31. 